\title{
Estimation of Genetic Variability and Character Association for Yield Characters in Blackgram (Vigna mungo (L.) Hepper)
}

\author{
K. Joshna*, G. R. Lavanya and M. Jemima Das \\ Department of Genetics and Plant Breeding, Naini Agricultural Institute, \\ SHUATS, Prayagraj (U.P.), India \\ *Corresponding author
}

\section{A B S T R A C T}

\section{Keywords}

Black gram, GCV, PCV, Variability, Heritability, Correlation, Path analysis

\section{Article Info}

\section{Accepted:}

07 January 2021

Available Online:

10 February 2021
The present investigation was carried out with a view to study the magnitude of variability, correlation and path analysis excluding reciprocals involving 25 genotypes in Black gram during Kharif - 2019. The experimental material was planted in randomized block design with three replications at the Field Experimentation Centre, Department of Genetics and Plant Breeding, SHUATS, Prayagraj, U.P. The analysis of variance indicated that parents were significant for all the characters, indicating presence of considerable amount of genetic variability in the germplasm tested. Number of clusters per plant, seed yield per plant exhibited high GCV, PCV and genetic parameters revealed that heritability (broad sense) and genetic advance as \% of mean values were high for days to maturity, harvest index, but in case of genetic advance and genetic advance as $\%$ of mean values were high for seed yield per plant indicating that selection would be fruitful for improvement of these traits. Correlation coefficient analysis revealed that seed yield per plant exhibited positive and significant association with Number of branches per plant, harvest index, Number of pods per plant, Number of seeds per pod. The Path coefficient analysis indicated that selection for days to maturity, Plant height, Number of clusters per plant, seed index, harvest index, would directly increase seed yield. The genotypes SHEKAR -3, IPU862 X IPU96-1, SHEKAR, and G.C 9120 X IC106194were observed as promising genotypes for importance quantitative traits.

\section{Introduction}

Black gram (Vigna mungo L. Hepper, 2n=22) is an important self-pollinated and short duration legume crop belonging to the family Leguminosae and sub-family Papilionaceae. India is the center of origin of black gram (Mehra et al., 2016). It is popularly known as "urd bean and mash" is an important short duration and self pollinated Kharif legume crop and is an important part of Indian diet. It is rich source of protein (25-28\%), carbohydrates (62-65\%), fiber(3.5- 4.5\%), ash (4.5-5.5\%), oil (0.5-1.5\%), amino acids like lysine, vitamins like thiamine, niacin, riboflavin and much needed iron and phosphorus (Sohel et al., 2016). Black gram was sown over an area of 50.31 lakh/ha and recorded a production of 32.84 lakh tonnes and yield level of $653 \mathrm{~kg} / \mathrm{ha}$ in India. Where 
as the total coverage under Black gram in Uttar Pradesh is 5.88 Lha with a production 3.05 Lt. and the productivity $520 \mathrm{~kg} / \mathrm{ha}$ (Directorate Pulse Development, Ministry of Agriculture \& Farmers Welfare-2018-19). The per capita availability of pulses has declined from $60.7 \mathrm{~g} /$ day in 1951 to $35.5 \mathrm{~g}$ day-1 in 2007 as against the FAO/WHO's recommendation of $80 \mathrm{~g} /$ day (Economic Survey, 2008-09). Blackgram has a wide range of economic value. It is well known that $50 \mathrm{~g}$ pulses/person/day should be consumed in addition to other sources of protein such as cereals, milk, meat and egg which is very difficult task to achieve as the production and productivity of pulse crops including the blackgram is very low. Hence, there is a strong need to improve the productivity of crop. This could be achieved by studying the genetic architecture of this crop for yield improvement.

\section{Materials and Methods}

\section{Description of the Study Area}

The present investigation was carried out at the Field Experimentation Centre, Department of Genetics and Plant Breeding, SHUATS, Prayagraj (U.P.) during Kharif, 2019. The University is situated on the left side of Prayagraj - Rewa National Highway, about 5 $\mathrm{km}$ away from Prayagraj city. All types of facilities necessary for cultivation of successful crop including field preparation, inputs and irrigation facilities were provided from the Department of Genetics and Plant Breeding, SHUATS, Prayagraj (U.P.). 2.2 Experimental Design and Procedure. The experiment was conducted in randomized block design with 25 genotypes which were replicated 3 times. Genotypes were randomly arranged in each replication divided into 75 plots. The gross area of experiment was $180 \mathrm{~m} 2$ and each plot size was $1 \times 1 \mathrm{~m}$. The row to row spacing was $30 \mathrm{~cm}$ and plant to plant distance was $10 \mathrm{~cm}$. 2.3 Data source and analysis. Data were recorded from 13 characters viz, days to $50 \%$ flowering, days to $50 \%$ pod setting, days to maturity, plant height $(\mathrm{cm})$, number of branches per plant, number of cluster per plant, number of pods per plant, pod length $(\mathrm{cm})$, number of seeds per pod, seed index $(\mathrm{g})$, biological yield $(\mathrm{g})$, harvest index and seed yield per plant $(\mathrm{g})$.

The 5 competitive plants from each of the replication were tagged and observations were taken from these tagged plants at various stages of the crop plant growth.

Mean values were computed and data were analysed for analysis of variance as suggested by (Fisher, 1936) and coefficient of variances as well as heritability (in broad sense), as suggested by Burton and Devane (1953). The estimates of genetic advance were obtained by the formula suggested by Lush (1949) and Johnson et al., (1955). Phenotypic and genotypic correlation and path coefficients of variation were computed as per the method given by Dewey and Lu(1959).

\section{Results and Discussion}

\section{Analysis of variance}

Mean sum of squares data for 13 characters were subjected to analysis of variance for experimental design.

The analysis of variance for different characters is presented in Table 1. The mean squares due to genotypes showed highly significant differences $(\alpha=0.01)$ for all characters which showed significant differences $(\alpha=0.05)$ indicating the presence of substantial amount of genetic variability among the Black gram genotypes. 
Table.1 Analysis of variance for 13 Quantitative characters in Black gram

\begin{tabular}{|c|c|c|c|c|}
\hline \multirow{2}{*}{ S. No } & Character & \multicolumn{3}{|c|}{ Mean sum of squares } \\
\cline { 3 - 5 } & Days to 50\% flowering & $\begin{array}{c}\text { Replication } \\
\text { (d.f.=2) }\end{array}$ & $\begin{array}{c}\text { Treatment } \\
\text { (d.f.=19) }\end{array}$ & $\begin{array}{c}\text { Error } \\
(\mathbf{d} . \mathbf{f}=\mathbf{3 8})\end{array}$ \\
\hline $\mathbf{1}$ & 24.3735 & $5.041^{* *}$ & $\mathbf{2 . 1 9 3}$ \\
\hline $\mathbf{2}$ & Days to 50\% Pod setting & 27.3735 & $4.83^{* *}$ & $\mathbf{2 . 1 2 3}$ \\
\hline $\mathbf{3}$ & Days to maturity & 0.2165 & $9.18^{* *}$ & $\mathbf{0 . 1 0 8}$ \\
\hline $\mathbf{4}$ & Plant height (cm) & 1754.24 & $73.58^{* *}$ & $\mathbf{2 4 . 2 3}$ \\
\hline $\mathbf{5}$ & Number of branches/plant & 0.9835 & $1.89^{* *}$ & $\mathbf{0 . 6 3 1}$ \\
\hline $\mathbf{6}$ & Number of clusters & 20.845 & $14.92^{* *}$ & $\mathbf{1 . 8 5}$ \\
\hline $\mathbf{7}$ & /plant & 43.8655 & $28.68^{* *}$ & $\mathbf{7 . 1 2 2}$ \\
\hline $\mathbf{8}$ & Number of pods /plant & 0.0195 & $0.071^{* *}$ & $\mathbf{0 . 0 1 5}$ \\
\hline $\mathbf{9}$ & Pod length (cm) & 0.459 & $0.69^{* *}$ & $\mathbf{0 . 2 3 5}$ \\
\hline $\mathbf{1 0}$ & Seed index (g) & 0.0225 & $0.13^{* *}$ & $\mathbf{0 . 0 4}$ \\
\hline $\mathbf{1 1}$ & Biological yield & 2.4155 & $14.38^{* *}$ & $\mathbf{2 . 4 8 9}$ \\
\hline $\mathbf{1 2}$ & Harvest Index & 8.8205 & $118.73^{* *}$ & $\mathbf{2 5 . 2 9 3}$ \\
\hline $\mathbf{1 3}$ & Seed yield / plant & $\mathbf{0 . 8 0 5}$ & $\mathbf{0 . 8 6} * *$ & $\mathbf{0 . 0 5}$ \\
\hline
\end{tabular}

* Significance at $5 \%$ level, $* *$ Significance at $1 \%$ level

Table.2 Genetic parameters for 13 Quantitative traits of Blackgram genotypes

\begin{tabular}{|c|c|c|c|c|c|c|}
\hline S.No & Character & GCV & PCV & $\mathbf{h}^{\mathbf{2}} \mathbf{b s}$ & GA & $\begin{array}{c}\text { GA as \% } \\
\text { mean }\end{array}$ \\
\hline $\mathbf{1}$ & Days to 50\% flowering & 2.166 & 3.94 & 30.216 & 1.103 & 2.453 \\
\hline $\mathbf{2}$ & Days to 50\% pod setting & 1.791 & 3.278 & 29.846 & 1.07 & 2.016 \\
\hline $\mathbf{3}$ & Days to maturity & 2.459 & 2.506 & 96.342 & 3.52 & 4.973 \\
\hline $\mathbf{4}$ & Plant height & 6.796 & 10.687 & 40.446 & 5.314 & 8.904 \\
\hline $\mathbf{5}$ & No. of branches/ plant & 7.161 & 11.328 & 39.962 & 0.844 & 9.326 \\
\hline $\mathbf{6}$ & No. of clusters/ plant & 17.475 & 20.857 & 70.195 & 3.602 & 30.16 \\
\hline $\mathbf{7}$ & No. of pods /plant & 13.567 & 19.144 & 50.221 & 3.913 & 19.805 \\
\hline $\mathbf{8}$ & No. of seeds / plant & 6.703 & 10.679 & 39.393 & 0.505 & 8.666 \\
\hline $\mathbf{9}$ & Pod length & 3.341 & 4.509 & 54.904 & 0.208 & 5.1 \\
\hline $\mathbf{1 0}$ & Seed index & 4.71 & 7.165 & 43.222 & 0.237 & 6.379 \\
\hline $\mathbf{1 1}$ & Biological yield & 13.564 & 17.307 & 61.422 & 3.214 & 21.899 \\
\hline $\mathbf{1 2}$ & Harvest index & 9.061 & 9.869 & 84.287 & 0.98 & 17.136 \\
\hline $\mathbf{1 3}$ & Seed yield / plant & 13.964 & 18.797 & 55.186 & 8.541 & 21.369 \\
\hline
\end{tabular}

VG= Genotypic variance VP = Phenotypic variance

$\mathrm{GCV}=$ Genotypic coefficient of variation $\mathrm{PCV}=$ Phenotypic coefficient of variation

$\mathrm{h}^{2}(\mathrm{bs})=$ Heritability broad sense $\mathrm{GA}=$ Genetic advance 
Table.3 Correlation coefficient between yield and its related traits in Blackgram genotypes at genotypic level

\begin{tabular}{|c|c|c|c|c|c|c|c|c|c|c|c|c|c|}
\hline Characters & $\begin{array}{c}\text { Days to } \\
50 \% \\
\text { flowering }\end{array}$ & $\begin{array}{c}\text { Days to } \\
50 \% \text { pod } \\
\text { setting }\end{array}$ & $\begin{array}{l}\text { Days to } \\
\text { Maturity }\end{array}$ & $\begin{array}{c}\text { Plant } \\
\text { height }\end{array}$ & $\begin{array}{l}\text { No. of } \\
\text { branche } \\
\text { s / plant }\end{array}$ & $\begin{array}{l}\text { No. of } \\
\text { clusters } \\
\text { / plant }\end{array}$ & $\begin{array}{l}\text { No of } \\
\text { pods / } \\
\text { plant }\end{array}$ & $\begin{array}{c}\text { No. of } \\
\text { seed /pod }\end{array}$ & $\begin{array}{c}\text { Pod } \\
\text { length }\end{array}$ & Seed index & $\begin{array}{c}\text { Biological } \\
\text { yield }\end{array}$ & $\begin{array}{c}\text { Harvest } \\
\text { Index }\end{array}$ & $\begin{array}{l}\text { Seed } \\
\text { yield/plant }\end{array}$ \\
\hline $\begin{array}{c}\text { Days to } \\
50 \% \\
\text { flowering }\end{array}$ & 1 & 0.136 & -0.163 & $0.513^{* *}$ & $0.228^{*}$ & $0.229^{*}$ & 0.085 & $0.252^{*}$ & $-0.752^{* *}$ & -0.045 & -0.213 & $-0.548^{* *}$ & -0.217 \\
\hline $\begin{array}{c}\text { Days to } \\
50 \% \text { pod } \\
\text { setting }\end{array}$ & & 1 & $0.845^{* *}$ & $0.250^{*}$ & $-0.249^{*}$ & $0.270^{*}$ & $0.273^{*}$ & 0.021 & $0.286^{*}$ & $0.275^{*}$ & 0.185 & 0.205 & 0.066 \\
\hline $\begin{array}{l}\text { Days to } \\
\text { Maturity }\end{array}$ & & & 1 & -0.020 & -0.055 & 0.207 & 0.027 & -0.020 & $0.512^{* *}$ & -0.037 & -0.087 & 0.115 & 0.201 \\
\hline $\begin{array}{c}\text { Plant } \\
\text { height }\end{array}$ & & & & 1 & $0.652^{* *}$ & -0.034 & 0.000 & 0.144 & $-0.416^{* *}$ & 0.169 & 0.093 & 0.122 & $-0.248 *$ \\
\hline $\begin{array}{c}\text { No. of } \\
\text { branches } \\
\text { /plant }\end{array}$ & & & & & 1 & $-0.250^{*}$ & -0.188 & 0.018 & $-0.226^{*}$ & $0.246^{*}$ & -0.157 & 0.213 & $0.285^{*}$ \\
\hline $\begin{array}{l}\text { No. of } \\
\text { cluster } \\
\text { s/plant }\end{array}$ & & & & & & 1 & $0.680^{* *}$ & $0.713^{* *}$ & 0.179 & 0.052 & 0.114 & 0.173 & $0.254^{*}$ \\
\hline $\begin{array}{c}\text { No of pods } \\
\text { /plant }\end{array}$ & & & & & & & 1 & $0.716^{* *}$ & 0.050 & $0.512^{* *}$ & $0.272^{*}$ & $0.367^{* *}$ & 0.037 \\
\hline $\begin{array}{l}\text { No. of seed } \\
\text { /pod }\end{array}$ & & & & & & & & 1 & 0.090 & 0.134 & 0.111 & $0.380^{* *}$ & $0.340^{* *}$ \\
\hline Pod length & & & & & & & & & 1 & 0.039 & 0.041 & 0.196 & 0.214 \\
\hline Seed index & & & & & & & & & & 1 & $0.375^{* *}$ & 0.203 & -0.151 \\
\hline $\begin{array}{l}\text { Biological } \\
\text { yield }\end{array}$ & & & & & & & & & & & 1 & $0.275^{*}$ & $0.735^{* *}$ \\
\hline $\begin{array}{c}\text { Harvest } \\
\text { Index }\end{array}$ & & & & & & & & & & & & 1 & $0.440^{* * *}$ \\
\hline $\begin{array}{l}\text { Seed yield } \\
\text { /plant }\end{array}$ & & & & & & & & & & & & & 1 \\
\hline
\end{tabular}


Table.4 Correlation coefficient between yield and its related traits in Blackgram genotypes at phenotypic level

\begin{tabular}{|c|c|c|c|c|c|c|c|c|c|c|c|c|c|}
\hline Characters & $\begin{array}{l}\text { Days to } \\
50 \% \\
\text { flowering }\end{array}$ & $\begin{array}{c}\text { Days to } \\
\mathbf{5 0 \%} \text { pod } \\
\text { setting }\end{array}$ & $\begin{array}{l}\text { Days to } \\
\text { Maturity }\end{array}$ & $\begin{array}{c}\text { Plant } \\
\text { height }\end{array}$ & $\begin{array}{c}\text { No. of } \\
\text { branches / } \\
\text { plant }\end{array}$ & $\begin{array}{l}\text { No. of } \\
\text { clusters } \\
\text { / plant }\end{array}$ & $\begin{array}{l}\text { No of pods } \\
\text { / plant }\end{array}$ & $\begin{array}{c}\text { No. of seed } \\
\text { /pod }\end{array}$ & Pod length & $\begin{array}{c}\text { Seed } \\
\text { index }\end{array}$ & $\begin{array}{c}\text { Biological } \\
\text { yield }\end{array}$ & $\begin{array}{l}\text { Harvest } \\
\text { Index }\end{array}$ & $\begin{array}{l}\text { Seed } \\
\text { yield/plant }\end{array}$ \\
\hline $\begin{array}{c}\text { Days to } \\
\mathbf{5 0 \%} \\
\text { flowering }\end{array}$ & 1 & -0.009 & -0.109 & 0.159 & $0.267^{*}$ & 0.107 & -0.047 & 0.030 & $-0.363^{* *}$ & 0.011 & $0.217^{*}$ & $-0.267^{*}$ & -0.037 \\
\hline $\begin{array}{c}\text { Days to } \\
\mathbf{5 0 \%} \text { pod } \\
\text { setting }\end{array}$ & & 1 & $0.425^{* *}$ & -0.029 & -0.113 & $0.251^{*}$ & 0.035 & -0.026 & $0.226^{*}$ & 0.001 & -0.021 & 0.181 & 0.161 \\
\hline $\begin{array}{c}\text { Days to } \\
\text { Maturity }\end{array}$ & & & 1 & -0.009 & -0.034 & 0.158 & $0.267^{*}$ & -0.047 & $0.367^{* *}$ & 0.003 & -0.072 & $0.271^{*}$ & 0.152 \\
\hline $\begin{array}{c}\text { Plant } \\
\text { height }\end{array}$ & & & & 1 & $0.332^{* *}$ & 0.008 & 0.085 & 0.081 & $0.262^{*}$ & 0.131 & 0.177 & $0.312^{* *}$ & -0.096 \\
\hline $\begin{array}{c}\text { No. of } \\
\text { branches } \\
\text { /plant }\end{array}$ & & & & & 1 & 0.084 & 0.167 & -0.244 & -0.040 & -0.277 & $0.251^{*}$ & 0.087 & $0.389^{* *}$ \\
\hline $\begin{array}{l}\text { No. of } \\
\text { cluste } \\
\text { rs/pla } \\
\text { nt }\end{array}$ & & & & & & 1 & $0.624^{* *}$ & $0.564^{* *}$ & $0.247^{*}$ & 0.044 & 0.180 & 0.153 & $0.419^{* *}$ \\
\hline $\begin{array}{c}\text { No of } \\
\text { pods/plant }\end{array}$ & & & & & & & 1 & $0.455^{* *}$ & 0.149 & $0.281^{*}$ & $0.259^{*}$ & $0.262^{*}$ & -0.027 \\
\hline $\begin{array}{c}\text { No. of seed } \\
\text { /pod }\end{array}$ & & & & & & & & 1 & 0.184 & $0.363^{* *}$ & 0.175 & $-0.343^{*}$ & $0.403^{* *}$ \\
\hline Pod length & & & & & & & & & 1 & -0.029 & $0.421^{* *}$ & $-0.413^{* *}$ & 0.014 \\
\hline Seed index & & & & & & & & & & 1 & $0.251^{*}$ & 0.107 & -0.128 \\
\hline $\begin{array}{c}\text { Biological } \\
\text { yield }\end{array}$ & & & & & & & & & & & 1 & 0.143 & $-0.808^{* * *}$ \\
\hline $\begin{array}{l}\text { Harvest } \\
\text { Index }\end{array}$ & & & & & & & & & & & & 1 & $0.443^{* *}$ \\
\hline $\begin{array}{c}\text { Seed yield } \\
\text { /plant }\end{array}$ & & & & & & & & & & & & & 1 \\
\hline
\end{tabular}


Table.5 Direct and indirect effects between yield and its different traits in Blackgram genotypes at genotypic level

\begin{tabular}{|c|c|c|c|c|c|c|c|c|c|c|c|c|c|}
\hline Characters & $\begin{array}{c}\text { Days to } \\
50 \% \\
\text { flowering }\end{array}$ & $\begin{array}{c}\text { Days to } \\
50 \% \text { pod } \\
\text { setting }\end{array}$ & $\begin{array}{l}\text { Days to } \\
\text { Maturity }\end{array}$ & $\begin{array}{l}\text { Plant } \\
\text { height }\end{array}$ & $\begin{array}{c}\text { No. of } \\
\text { branches / } \\
\text { plant }\end{array}$ & $\begin{array}{c}\text { No. of } \\
\text { clusters / } \\
\text { plant }\end{array}$ & $\begin{array}{c}\text { No of pods / } \\
\text { plant }\end{array}$ & $\begin{array}{c}\text { No. of seed } \\
\text { /pod }\end{array}$ & Pod length & Seed index & $\begin{array}{c}\text { Biological } \\
\text { yield }\end{array}$ & $\begin{array}{c}\text { Harvest } \\
\text { Index }\end{array}$ & $\begin{array}{l}\text { Seed } \\
\text { yield/plan } \\
\text { t }\end{array}$ \\
\hline $\begin{array}{c}\text { Days to } \\
50 \% \\
\text { flowering }\end{array}$ & -0.4987 & -0.17244 & -0.24187 & 0.63912 & -0.2967 & 0.11538 & -0.06361 & 0.1528 & 1.0095 & -0.0503 & 0.31385 & 0.12439 & -0.217 \\
\hline $\begin{array}{l}\text { Days to } \\
50 \% \text { pod } \\
\text { setting }\end{array}$ & -0.20408 & -0.2663 & 0.25448 & 0.31126 & 0.32402 & 0.1362 & -0.2035 & 0.0129 & -0.38352 & 0.30971 & -0.27163 & 0.04645 & 0.066 \\
\hline $\begin{array}{l}\text { Days to } \\
\text { Maturity }\end{array}$ & 0.24429 & -0.07056 & 0.48388 & -0.0251 & 0.07102 & 0.10453 & -0.02041 & -0.01216 & -0.68642 & -0.04217 & 0.12825 & 0.0261 & 0.201 \\
\hline $\begin{array}{c}\text { Plant } \\
\text { height }\end{array}$ & -0.76879 & -0.31636 & -0.02989 & 0.2459 & -0.8488 & -0.0171 & -0.00032 & 0.08732 & 0.55801 & 0.19033 & -0.13624 & 0.02768 & -0.248 \\
\hline $\begin{array}{l}\text { No. of } \\
\text { branches } \\
\text { /plant }\end{array}$ & -0.34141 & 0.315 & -0.0809 & 0.81183 & -0.303 & -0.1259 & 0.13981 & 0.01117 & 0.30279 & 0.27635 & 0.23057 & 0.04828 & 0.285 \\
\hline $\begin{array}{l}\text { No. of } \\
\text { cluster } \\
\text { s/plant }\end{array}$ & -0.34259 & -0.3417 & 0.30731 & -0.0423 & 0.325 & 0.5048 & -0.50631 & 0.43238 & -0.24059 & 0.05895 & -0.16783 & 0.03925 & 0.254 \\
\hline $\begin{array}{c}\text { No of } \\
\text { pods/plant }\end{array}$ & -0.12808 & -0.34625 & 0.0407 & 0.00054 & 0.24469 & 0.34339 & -0.74426 & 0.43418 & -0.06671 & 0.57589 & -0.40023 & 0.08334 & 0.037 \\
\hline $\begin{array}{c}\text { No. of seed } \\
\text { /pod }\end{array}$ & -0.37788 & -0.02695 & -0.02978 & 0.17953 & -0.024 & 0.36014 & -0.53322 & 0.60601 & -0.12109 & 0.15083 & -0.16363 & 0.08626 & 0.34 \\
\hline Pod length & 0.12776 & -0.36202 & 0.75925 & -0.5182 & 0.294 & 0.09052 & -0.03701 & 0.0547 & -0.3415 & 0.04392 & 0.05821 & 0.04455 & 0.214 \\
\hline Seed index & 0.06697 & -0.34845 & -0.0556 & 0.21069 & -0.3198 & 0.02644 & -0.3808 & 0.08121 & -0.05234 & 0.12555 & -0.55128 & 0.04597 & -0.151 \\
\hline $\begin{array}{l}\text { Biological } \\
\text { yield }\end{array}$ & 0.31974 & -0.23382 & -0.12936 & 0.11539 & 0.20416 & 0.05759 & -0.20248 & 0.06741 & 0.05308 & 0.42178 & 0.47111 & 0.06235 & 0.735 \\
\hline $\begin{array}{c}\text { Harvest } \\
\text { Index }\end{array}$ & 0.8213 & -0.25912 & 0.17063 & 0.15194 & -0.277 & 0.08728 & -0.27325 & 0.23029 & -0.26331 & 0.22794 & -0.40407 & 0.22699 & 0.44 \\
\hline
\end{tabular}

* Significance at $5 \%$ level, ** Significance at $1 \%$ level Residual effect 0.06678 
Table.6 Direct and indirect effects between yield and its different traits in Blackgram genotypes at phenotypic level

\begin{tabular}{|c|c|c|c|c|c|c|c|c|c|c|c|c|c|}
\hline Characters & $\begin{array}{c}\text { Days to } 50 \% \\
\text { flowering }\end{array}$ & $\begin{array}{c}\text { Days to } \\
50 \% \text { pod } \\
\text { setting }\end{array}$ & $\begin{array}{l}\text { Days to } \\
\text { Maturity }\end{array}$ & $\begin{array}{c}\text { Plant } \\
\text { height }\end{array}$ & $\begin{array}{l}\text { No. of } \\
\text { branche } \\
\text { s / plant }\end{array}$ & $\begin{array}{c}\text { No. of } \\
\text { clusters / } \\
\text { plant }\end{array}$ & $\begin{array}{l}\text { No of } \\
\text { pods / } \\
\text { plant }\end{array}$ & $\begin{array}{l}\text { No. of seed } \\
\text { /pod }\end{array}$ & $\begin{array}{c}\text { Pod } \\
\text { length }\end{array}$ & Seed index & $\begin{array}{c}\text { Biological } \\
\text { yield }\end{array}$ & $\begin{array}{c}\text { Harvest } \\
\text { Index }\end{array}$ & $\begin{array}{l}\text { Seed } \\
\text { yield/plant }\end{array}$ \\
\hline $\begin{array}{l}\text { Days to } 50 \% \\
\text { flowering }\end{array}$ & -0.04378 & -0.00034 & -0.00037 & 0.00304 & 0.00091 & 0.00073 & -0.00334 & -0.00102 & 0.00115 & 0.00028 & 0.14968 & -0.14411 & -0.037 \\
\hline $\begin{array}{l}\text { Days to } 50 \% \\
\text { pod } \\
\text { setting }\end{array}$ & 0.00039 & 0.0381 & 0.00145 & -0.0006 & 0.00152 & 0.00086 & 0.00251 & 0.0009 & -0.00072 & 0.00001 & 0.01949 & 0.09745 & 0.161 \\
\hline $\begin{array}{l}\text { Days to } \\
\text { Maturity }\end{array}$ & 0.00479 & 0.01619 & 0.0034 & -0.0002 & 0.00046 & 0.00108 & 0.00077 & 0.00161 & -0.00116 & 0.00008 & 0.0659 & 0.05929 & 0.152 \\
\hline Plant height & -0.00697 & -0.0011 & -0.00003 & 0.0191 & -0.0045 & 0.00006 & 0.00604 & -0.00278 & 0.00038 & 0.00327 & -0.16162 & 0.05192 & -0.096 \\
\hline $\begin{array}{l}\text { No. of } \\
\text { branches } \\
\text { /plant }\end{array}$ & 0.00297 & -0.00432 & -0.00012 & 0.00635 & -0.013 & 0.00058 & 0.0118 & -0.00042 & 0.00013 & 0.00272 & -0.00788 & 0.04709 & 0.389 \\
\hline $\begin{array}{l}\text { No. of } \\
\text { clusters/p } \\
\text { lant }\end{array}$ & -0.00468 & 0.00477 & 0.00054 & 0.00015 & -0.0011 & 0.0068 & 0.04415 & -0.01923 & -0.00078 & 0.0011 & -0.16408 & 0.08249 & 0.419 \\
\hline $\begin{array}{l}\text { No of } \\
\text { pods/plant }\end{array}$ & 0.00206 & 0.00135 & 0.00004 & 0.00163 & -0.0022 & 0.00427 & 0.07077 & -0.01553 & -0.00047 & 0.00703 & -0.23701 & 0.14119 & -0.027 \\
\hline $\begin{array}{l}\text { No. of seed } \\
\text { /pod }\end{array}$ & -0.00131 & -0.00101 & -0.00016 & 0.00156 & -0.0002 & 0.00386 & 0.03222 & -0.0341 & -0.00058 & 0.00187 & -0.15973 & 0.11619 & 0.403 \\
\hline Pod length & 0.01591 & 0.00863 & 0.00125 & -0.0023 & 0.00054 & 0.00169 & 0.01053 & -0.00627 & -0.0032 & -0.00072 & -0.06367 & 0.0518 & 0.014 \\
\hline Seed index & -0.00049 & 0.00002 & 0.00001 & 0.0025 & -0.0015 & 0.0003 & 0.01986 & -0.00254 & 0.00009 & 0.02506 & -0.22947 & 0.05786 & -0.128 \\
\hline Biological yield & 0.00717 & -0.00081 & -0.00025 & 0.00338 & -0.0001 & 0.00123 & 0.01836 & -0.00596 & -0.00022 & 0.00629 & -0.91376 & 0.07698 & -0.808 \\
\hline Harvest Index & 0.0117 & 0.00688 & 0.00037 & 0.00184 & -0.0012 & 0.00105 & 0.01852 & -0.00734 & -0.0003 & 0.00269 & -0.13038 & 0.53948 & 0.443 \\
\hline
\end{tabular}

* Significance at 5\% level, ** Significance at $1 \%$ level Residualeffect 0.02103 


\section{Parameters of genetic variability}

The parameters of genetic variability such as mean, range, phenotypic coefficient of variation(pcv), genotypic coefficient of variation $(\mathrm{GCV})$,heritability, genetic advance and genetic advance as percent of mean were estimated for all quantitative characters are presented in table 2. The Phenotypic coefficient of variation (PCV) ranged from 2.50 (days to maturity) to (20.85) number of clusters per plant. High PCV were recorded for number of clusters per plant (20.85). Low PCV were recorded for seed index (7.16), pod length (4.50), days to $50 \%$ flowering (3.94), days to $50 \%$ podsetting (3.27), days to maturity (2.50). Similar results are reported by Gowsalya et al., (2016), Hemalatha et al., (2017). Genotypic coefficient of variation ranged from days to $50 \%$ of podsetting (1.79) to number of clusters per plant (17.47). High GCV were recorded for number of clusters per plant (17.47).

Low GCV were recorded for number of seeds per plant (6.70), followed by seed index (4.71), pod length (3.34), days to maturity (2.45), days to $50 \%$ of flowering (2.166), and days to $50 \%$ of podsetting (1.79). Similar results are reported by Kumar et al., (2015), Gowsalya et al., (2016), and Hemalatha et al., (2017).

\section{Heritability}

The estimate of heritability (\%) in the broad sense for 13 charters studied which range from 29.84 (days to $50 \%$ of pod setting) to96.34 (days to maturity). The highest heritability (broadsense) were recorded for characters i.e. days to maturity (96.34), harvest index (84.28), number of clusters per plant (70.19), and biological yield (61.42). The lowest heritability (broadsense) were recorded for characters plant height (40.44), number of branches per plant (39.96), number of seeds per plant (39.39), days to $50 \%$ of flowering (30.21), and days to $50 \%$ of pod setting (29.84). Similar results are reported by Gowsalya et al., (2016), Patel et al., (2014).

\section{Genetic advance (\%)}

The genetic advance $(\%)$ which ranged from 8.54 (seed yield per plant) to 0.208 (pod length). The highest genetic advance were recorded for seed yield per plant (8.54) followed by plant height (5.31), number of pods perplant (3.91), number of clusters per plant (3.60), days to maturity (3.52). The lowest genetic advance were recorded for harvest index (0.98), number of branches per plant (0.84), number of seeds per plant $(0.50)$, pod length (0.23), and pod length (0.208).Similar results are reported by Priya et al.(2018).

\section{Genetic advance as \% of mean}

The genetic advance as percent of mean varied from 30.16 (number of clusters per plant) to 2.0 (days to $50 \%$ of pod setting). The highest genetic advance as \% of mean were recorded for no of clusters per plant (30.16) followed by biological yield (21.8), seed yield per plant (21.36), number of pods per plant (19.8) and harvest index (17.13).The lowest genetic advance as $\%$ of mean were recorded for days to maturity (4.9), days to $50 \%$ of flowering (2.4) and days to $50 \%$ of pod setting (2.0) Similar results are reported by Gowsalya et al., (2016), Patel et al., (2014), Punia et al.,(2014).

\section{Correlation coefficient analysis}

The correlation coefficient is a statistical measure which is used to find out the degree (strength) and direction of the relationship between two or more variable components (Panigrahi et al., 2014). 


\section{Correlation with seed yield}

Correlation coefficient analysis among grain yield and its contributing characters are shown in table 3 and table 4. Genotypic correlation coefficient analysis revealed that seed yield per plant showed highly significant and positive association with biological yield $\left(0.735^{* *}\right)$, harvest index $(0.440 * *)$, number of seeds per plant $\left(0.340^{* *}\right)$, number of branches per plant $\left(0.285^{*}\right)$, number of clusters per plant $(0.254 *)$, and negative significant association with plant height $\left(-0.248^{*}\right)$. It also showed positive but non-significant association with Days to $50 \%$ of pod setting (0.066), number of pods per plant (0.037), pod length (0.214), days to maturity $(0.201)$. While negative but non-significant association was recorded for Days to50\% flowering (- 0.217), seed index (-0.151). Similar results are reported by Rajasekhar et al., (2017), Mehra et al., (2016), Kumar et al., (2015). Phenotypic correlation coefficient analysis revealed that Seed yield per plant showed highly significant and positive association with harvest index $(0.443 * *)$, number of seeds per plant $\left(0.403^{* *}\right)$, number of clusters per plant $\left(0.419^{* *}\right)$, number of branches per plant $(0.389 * *)$. Positive nonsignificant association with pod length (0.014), days to maturity (0.152), days to $50 \%$ of pod setting (0.161). Negative significant association with biological yield $\left(-0.808^{* *}\right)$. Negative non-significant association with seed index (-0.128), number of pods per plant (- 0.027), plant height (-0.096), days to $50 \%$ of flowering $(-0.037)$, these findings were in accordance with Rajasekar et al., (2017), Mehra et al.(2016), Kumar et al (2015), Punia et al., (2014), Bharti et al., (2014).

\section{Path coefficient analysis}

Path coefficient analysis is an efficient statistical technique specially designed to qualify the interrelationship of different components and their direct and indirect effects on yield (Pushpa et al., 2013).

\section{Seed yield with other yield traits}

Genotypic path coefficient analysis revealed that character seed yield per plant showed positive direct effect with Number of seeds per pod (0.60) followed by number of clusters per plant (0.540), days to maturity (0.48),plant height $(0.24)$,harvest index $(0.22)$ and seed index $(0.12)$ where as it showed negative direct effect with days to $50 \%$ of pod setting $(-0.26)$ followed by pod length ($0.34)$ number of branches per plant $(-0.30)$, biological yield (-0.47) days to $50 \%$ of flowering (-0.49), and number of pods per plant (-0.74). Aijaz et al., (2002),Singh et al., (2009), Muzibul et al.(2010), and Reni et al., (2013). Phenotypic path coefficient analysis revealed that character seed yield per plant showed positive direct effect with harvest index (0.539)followed by number of pods per plant $(0.070)$, days to $50 \%$ of pod setting (0.038), days to maturity (0.0034), seed index (0.025), plant height (0.019) and number of clusters per plant (0.006). Whereas it showed negative direct effect with Biological yield ($0.91)$ followed by days to $50 \%$ of flowering ($0.04)$, number of branches per plant (-0.013), number of seeds per pod (-0.034), and pod length (-0.003) Rajasekhar et al., (2017) Baudhbharti et al., (2014), Mishra et al., (2014) (Table 5 and 6).

From the present investigation it is concluded that analysis of variation showed significant variation to all the characters. Among 25 genotypes of black gram on the basis of mean performance SHEKAR -3 followed by IPU862 X IPU96-1, SHEKAR and G.C 9120 $\mathrm{X}$ IC 106194 was found to be superior and showed possessed maximum seed yield.

Number of clusters per plant, seed yield per plant exhibited high GCV, PCV and Genetic 
parameters revealed that heritability (broad sense) were high for days to maturity, harvest index, but in case of genetic advance and genetic advance as \% of mean values were high for seed yield per plant. High estimates of heritability coupled with high genetic advance as percent of mean recorded for number of clusters per plant and followed by biological yield.

Correlation coefficient analysis revealed that with number of branches per plant, harvest index, number of pods perplant, number of seeds per pod, exhibited positive significant association with seed yield per plant at both genotypic and phenotypic levels.

Path analysis revealed that the characters days to maturity, Plant height, number of clusters per plant, seed index, harvest index, exhibited positive direct effect on seed yield at both phenotypic and genotypic levels.

All four genotypes mentioned above with these characters can be used for further improvement and development of blackgram.

\section{Acknowledgement}

We are thankful to the Honorable Vice Chancellor, HOD, Teaching and nonTeaching staff of the Department of Genetics and Plant Breeding, Naini Agricultural Institute, Sam Higginbottom University of Agriculture, Technology and Sciences, Prayagraj-211007, U.P., for providing all necessary facilities and support.

\section{References}

Ali, M.N., Gupta Suparna, Bhattacharyya Somnath and Sarkar, H.K. (2008). Evaluation of black gram (Vigna mungo (L.) Hepper) germplasm using multivarietal analysis journal of Environment and Ecology, 26(2): 943-945.
Bakshi, A., Bala, S. and Dostisder, K.G. (2006). Character association for seed yield components in blackgram (Vigna mungo (L). Hepper) journal of Environment and Ecology, 24(3): 943945.

Bharti, Singh, S. and Kumar, P. (2014). Study on correlation and path analysis in blackgram (Vigna mungo (L.) Hepper). Journal of Legume Research, 18 (2): 25-28.

Chauhan, M.P., Mishra A.C. and Singh A.K. (2007).Correlation and path analysis in urd bean. Journal of LegumeResearch 30(3): 205-208.

Dadepeer, P., Ravikumar, R.L. and Salimath, P.M. (2009). Genetic variability and character association in local greengram (Vigna radiata (L.)Wilczek.) genotypes of Karnataka. Journal of Environment and Ecology, 27(1): 165-169.

Deepshikha, Lavanya, G.R. and Kumar, S. (2014). Assessment of Genetic variability for Yield and Its Contributing Traits in Blackgram (Vigna mungo (L.) Hepper). journal of Trends in Biosciences, 7(18) : 28352838 Dewey, D. and Lu, K.H. (1959). A correlation and path coefficient analysis of components of crested wheat grass seed production. Agronomy Journal, 51: 515-518.

Falconer, D.S. (1981). Introduction to Quantitative genetics, 3rd ed. Longman, New York. Pp. 340.

Fisher, R.A. (1918). The correlation between relatives of the supposition of Mendelian inheritance. Journal of France Royal Society of Edinburgh, 52: 399-433.

Gowsalya, P., Kumaresan, D. Packiaraj, D. and Bapu, K.J.R. (2016). Genetic variability and character association for biometrical traits in blackgram (Vigna mungo L. Hepper). Electronic Journal of Plant Breeding. 7(2): 317-324. 
Hemalatha, K., Sapna, S.L. and Gabrial, M.L. (2017). Study on Genetic Variability and Correlation in Blackgram (Vigna mungo L. Hepper). Journal of Pharmacognosy and Phytochemistry, 6(4): 674-676

Isha Parveen, S., Reddy Sekhar, M., Mohan Reddy, D. and Sudhakar, P. (2011).Correlation and path coefficient analysis for yield and yield components in blackgram (Vigna mungo (L.) Hepper). International Journal of Applied Biology and Pharmaceutical Technology, 2(3): 619-625.

Jayamani, P. and Sathya. (2013). Genetic diversity in pod characters in blackgram. Journal of Legume Research, 36(3): 220-223.

Jyothsna, S., Patro, T.S.S.K., Ashok, S. Sandhya Y., Rani and Neeraja, B (2016). Character association and Path analysis of Seed Yield and its Yield Components in Black gram (Vigna mungo (L.) Hepper). International Journal of Theoretical \&Applied Sciences, 8(1): 12-16.

Konda, C.R., Salimath, P.H. and Mishra, M.N. (2008). Correlation and path coefficient analysis in blackgram (Vigna mungo (L.) Hepper). Journal of Legume Research 31(3): 202-205.

Konda, C., Salimath, P. and Mishra, M. (2008). Correlation and path coefficient analysis in black -gram (Vigna mungo (L.) Hepper). Legume Research-An International Journal, 31(3) : 202-205.

Kumar, G. V., Vanaja, M., Lakshmi, N. J and Maheswari, M. (2015). Studies on variability, heritability and genetic advance for quantitative traits in blackgram (Vigna mungo (L.) Hepper). Agricultural Research Journal, 52(4) : 28-31.

Loganathan, P., Sarvanan, K. and Ganesan, J. (2001). Genetic variability in greengram Journal of .Research Crops, 2(3) : 396-
397.

Mehra, Reena, Tikle, A.N., Saxena, Ashok., Munjal, Ashok., Rekhakhandia and Singh, Mahipal (2016). Correlation, path-coefficient and genetic diversity in Blackgram (Vigna mungo L. Hepper). International Research Journal of Plant Science, 7(1): 001-011.

Panigrahi, K.K. and Baisakh, B. (2015). Correlation analysis for quantitative traits in blackgram (Vigna mungo (L.) Hepper) in different seasons. International Journal of Scientific and Research publications, 5, Issue 4.

Punia, S., Gautam, N., Ram, B., Verma, P., Dheer, M., Jain, N.K and Jat, V.S. (2014). Genetic variability and correlation studies in urd-bean (Vigna mungo L. Hepper). Journal of Legume Research, 37(6):580- 584.

Parameswarappa, S.G. and Lamani, K.D. (2005). Genetic estimates, association and path coefficient analysis in blackgram (Vigna mungo (L.) Hepper). Karnataka Journal of Agricultural Sciences, 18(1): 21-23.

Parveen, S.I., Sekhar, M.R., Reddy, D.M. and Sudhakar, P. (2011). Correlation and path coefficient analysis for yield and yield components in blackgram (Vigna mungo(L.)Hepper). International Journal of Applied Biology and Pharmaceutical Technology, 2(3):619625.

Pradhan, K.C. and Misra, P.K. (2005). Character association in blackgram (Vigna mungo (L.) Hepper).Journal of Environment and Ecology ,23(4) : 737742.

Rahim, M.A., Mia, A.A. Mahmud, F. Zeba N. and Afrin. K.S. (2010). Genetic variability, character association and genetic divergence in mung bean (Vigna radiata L. Wilczek). Pakistan orthodontic Journal, 3(1): 16.

Rajan, R.E., Wilson, D. and Kumar, V. 
(2001). Correlation and path analysis of blackgram (Vigna mungo(L.) Hepper). Madras Agriculture Journals, 87(10/12) : 590-593.

Rajasekhar,D., Sapna, S.L. and Gabrial, M.L. (2017).Character association and path analysis for seed yield and its components in blackgram [Vigna mungo (L.) Hepper]. Journal of Plant Archives, 17(1): 467- 471.

Rao, M., Rao Y.K. and Reddy M.V. (2006). Evaluation of mung bean germplasm for yield and yield components. Journal of Legume Research, 29(1): 73-75.

Singh, I.P., Kumar, A., Singh, J.D. and. Singh, K.P. (2007). Genetic variation, character association and path analysis between grain yield and its component in blackgram (Vigna mungo (L.) Hepper) International Research Journal of Plant Science, 7(1): 001-011.

Sreelakshmi, C and Reddy Sekhar, M. (2011). Comparison between correlation and path analysis studies in the full sib progenies and F3 bulk population among yield and its attributes in two crosses of greengram (Vigna radiata (L.) Wilczek). Electronic Journal of
Plant Breeding, 2(2): 258-262.

Umadevi, M. and Ganesan, N.M. (2007). Variability, correlation and path analysis for yield and yield components in blackgram (Vigna mungo (L.) Hepper). Journal of Crop Research, 31(2) : 253-257.

Veeramani, N., Venkatesan, M. Thangavel P. and Ganesan J. (2005). Genetic variability, heritability and genetic advance analysis in segregating generation of black gram (Vigna mungo (L.) Hepper). Journal of Legume Research, 28(1): 49 - 51.

Vijiyalaxmi and Bhattacharya A. (2006). Mung bean seed yield: III. Effect of yield attributing traits and phenology. Journal of Legume Research, 29(1): 1117.

Wani, S.K., Sridevi, O. and Salimath, P.M. (2007). Studies on genetic variability correlation and path analysis in chilli (capsicum annum). Journal of Annals of Biological Sciences, 23(2 ): 117-121.

Zukovskiji, P.M. (1962). Cultivated plants and their wild relatives, Commonwealth Agriculture Bureau, London.

\section{How to cite this article:}

Joshna, K., G. R. Lavanya and Jemima Das, M. 2021. Estimation of Genetic Variability and Character Association for Yield Characters in Blackgram (Vigna mungo (L.) Hepper). Int.J.Curr.Microbiol.App.Sci. 10(02): 836-847. doi: https://doi.org/10.20546/ijcmas.2021.1002.099 